J. Austral. Math. Soc. (Series A) 12 (1987), 390-398

\title{
GENERALIZATIONS OF F. E. BROWDER'S SHARPENED FORM OF THE SCHAUDER FIXED POINT THEOREM
}

\author{
KOK-KEONG TAN \\ (Received 18 June 1985; revised 6 February 1986) \\ Communicated by J. H. Rubinstein
}

\begin{abstract}
Let $E$ be a Hausdorff topological vector space, let $K$ be a nonempty compact convex subset of $E$ and let $f, g: K \rightarrow 2^{E}$ be upper semicontinuous such that for each $x \in K, f(x)$ and $g(x)$ are nonempty compact convex. Let $\Omega \subset 2^{E}$ be convex and contain all sets of the form $x-f(x), y-x+g(x)-$ $f(x)$, for $x, y \in K$. Suppose $p: K \times \Omega \rightarrow \mathbf{R}$ satisfies: (i) for each $(x, A) \in K \times \Omega$ and for $\varepsilon>0$, there exist a neighborhood $U$ of $x$ in $K$ and an open subset set $G$ in $E$ with $A \subset G$ such that for all $(y, B) \in K \times \Omega$ with $y \in U$ and $B \subset G,|p(y, B)-p(x, A)|<\varepsilon$, and (ii) for each fixed $x \in K$, $p(x, \cdot)$ is a convex function on $\Omega$. If $p(x, x-f(x)) \leqslant p(x, g(x)-f(x))$ for all $x \in K$, and if, for each $x \in K$ with $f(x) \cap g(x)=\varnothing$, there exists $y \in K$ with $p(x, y-x+g(x)-f(x))<$ $p(x, x-f(x))$, then there exists an $x_{0} \in K$ such that $f\left(x_{0}\right) \cap g\left(x_{0}\right) \neq \varnothing$. Anotber coincidence theorem on a nonempty compact convex subset of a Hausdorff locally convex topological vector space is also given.
\end{abstract}

1980 Mathematics subject classification (Amer. Math. Soc.): 47 H 10, 54 C 60.

Keywords and phrases: fixed point, Schauder fixed point theorem, set-value map, lower semicontinuous, upper semicontinuous, ultimately continuous, convex function, inward set, outward set, topological vector space, locally convex topological vector space.

\section{Introduction and preliminaries}

The classical Schauder fixed point theorem asserts that every continuous self-map of a nonempty compact convex subset of a Banach space has a fixed point. Obviously the Schauder fixed point theorem cannot be extended to non-self-maps without additional conditions. Many generalizations for single- or multi-valued

This work was partially supported by NSERC of Canada under grant A-8096.

(C) 1987 Australian Mathematical Society 0263-6115/87 \$A2.00+0.00 
maps have been obtained, for example see [2], [3], [5], [7] and [8]. Recently, F. E. Browder [4] gave a rather sharp improvement of these results for single-valued maps. Generalizations of those results in [4] to set-valued maps are obtained by $\mathbf{S}$. Reich $[12,13]$, J. H. Jiang $[9,10]$ and others. In this paper, we shall extend some of Browder's results in [4] to set-valued maps in different directions, one of which extends a result of $S$. Reich in [12].

We shall denote by $\mathbf{R}$ the real line and, for any nonempty set $X$, by $2^{X}$ the collection of all nonempty subsets of $X$. Now let $X$ and $Y$ be topological spaces. Then a map $f: X \rightarrow 2^{Y}$ is said to be (i) lower semicontinuous (respectively, upper semicontinuous) [1] at $x_{0} \in X$ if for each open set $G$ in $Y$ with $G \cap f\left(x_{0}\right) \neq \varnothing$ (respectively, with $f\left(x_{0}\right) \subset G$ ), there is a neighborhood $U$ of $x_{0}$ in $X$ such that $G \cap f(x) \neq \varnothing$ (respectively, $f(x) \subset G$ ) for all $x \in U$; (ii) lower semicontinuous (respectively, upper semicontinuous) on $X$ if $f$ is lower semicontinuous (respectively, upper semicontinuous) at each point of $X$; (iii) continuous on $X$ if $f$ is both lower semicontinuous on $X$ and upper semicontinuous on $X$. Also if $\Omega \subset 2^{Y}$, then a map $p: X \times \Omega \rightarrow \mathbf{R}$ is said to be (iv) ultimately continuous at $(x, A)$ if for each $\varepsilon>0$, there exist a neighborhood $U$ of $x$ in $X$ and an open set $G$ in $Y$ with $A \subset G$ such that $|p(y, B)-p(x, A)|<\varepsilon$ for all $(y, B) \in X \times \Omega$ with $y \in U$ and $B \subset G$; (v) ultimately continuous on $X \times \Omega$ if $p$ is ultimately continuous at each point of $X \times \Omega$. We note that in the case $\Omega=\{\{y\}: y \in Y\}$, if we write $p(x, y)=p(x,\{y\})$, then the notions of ultimate continuity and continuity coincide. If $A \subset X, \operatorname{cl}(A)$ denotes the closure of $A$ in $X$. Next let $E$ be a vector space, let $K$ be a nonempty subset of $E$ and let $x \in K$; then the inward set and outward set [8] of $K$ at $x$, denoted by $I_{K}(x)$ and $O_{K}(x)$, respectively, are defined by

$$
I_{K}(x)=\{y \in E: \text { there exist } u \in K \text { and } r>0 \text { such that } y=x+r(u-x)\}
$$

and

$$
O_{K}(x)=\{y \in E \text { : there exist } u \in K \text { and } r>0 \text { such that } y=x-r(u-x)\} \text {. }
$$

Also, a subset $\Omega$ of $2^{E}$ is convex if for each $A, B \in \Omega$ and for each $t \in[0,1]$, $t A+(1-t) B \in \Omega$. Moreover, if $E$ is a topological vector space, we shall denote by $\mathscr{X}(E)$ the collection of all compact convex sets in $2^{E}$ and by $\mathscr{C}(E)$ the collection of all closed convex sets in $2^{E}$. Finally we shall need the following fixed point theorem of K. Fan [6]:

TheOREM (K. Fan [6]). Let $K$ be a nonempty compact convex subset of a Hausdorff topological vector space $E$ and let $S: K \rightarrow 2^{K}$. Suppose, for each $x \in K$, that $S(x)$ is convex, while for each $u \in K$, the set $S^{-1}(u)=\{y \in K: u \in S(y)\}$ is open in $K$. Then there exists $x_{0} \in K$ such that $x_{0} \in S\left(x_{0}\right)$. 


\section{Main results}

The following two propositions are easy consequences of the definitions.

Proposition 2.1. Let $E$ be a topological vector space, let $K \subset E$ be nonempty, let $f, g: K \rightarrow 2^{E}$ be lower semicontinuous, let $h: K \rightarrow 2^{E}$ be upper semicontinuous and let $c \in \mathbb{R}$. Then $f+g$ and $c g$ are lower semicontinuous, and ch is upper semicontinuous.

Proposition 2.2. Let $E$ be a topological vector space, let $K \subset E$ be nonempty and let $f, g: K \rightarrow 2^{E}$ be upper semicontinuous such that for each $x \in K, f(x)$ and $g(x)$ are both compact. Then $f+g$ is also upper semicontinuous.

We note that Proposition 2.2 is false if the condition " $f, g: K \rightarrow 2^{E}$ be upper semicontinuous such that for each $x \in K, f(x)$ and $g(x)$ are both compact" is replaced by the condition " $f, g: K \rightarrow \mathscr{C}(E)$ be upper semicontinuous such that for each $x \in K$, at least one of $f(x)$ and $g(x)$ is compact." This can be seen from the following:

EXAMPLE 2.3. Let $E=\mathbb{R}^{2}$ and let $K=\left\{(x, y) \in \mathbb{R}^{2}: x^{2}+y^{2} \leqslant 1\right.$ and $x$, $y>0$. Define $f: K \rightarrow \mathscr{K}(E)$ by

$$
f(r \cos \theta, r \sin \theta)=\{(t \cos \theta, t \sin \theta): r \leqslant t \leqslant 2\}
$$

for each $r \in(0,1]$ and $\theta \in(0, \pi / 2)$. Define $g: K \rightarrow \mathscr{C}(E)$ by

$$
g(x, y)=\{(z, 0): z \geqslant x\}
$$

for all $(x, y) \in K$. It can be easily checked that $f$ and $g$ are both upper semicontinuous (in fact, both continuous) but $f+g$ is not upper semicontinuous.

The following result generalizes Proposition 2 in [4] and also Theorem 1 in [7] to set-valued maps.

Theorem 2.4. Let $E$ be a Hausdorff topological vector space, let $K \subset E$ be nonempty compact convex and let $f, g: K \rightarrow \mathscr{K}(E)$ be upper semicontinuous. Let $\Omega \subset 2^{E}$ be convex and contain all sets of the form $x-f(x), y-x+g(x)-f(x)$, for $x, y \in K$. Suppose $p: K \times \Omega \rightarrow \mathbb{R}$ is ultimately continuous such that for each $x \in K, p(x, \cdot)$ is a convex function on $\Omega$. Assume that

(i) $p(x, x-f(x)) \leqslant p(x, g(x)-f(x))$ for all $x \in K$, and

(ii) for each $x \in K$ with $f(x) \cap g(x)=\varnothing$, there exists $y \in K$ such that $p(x, y-x+g(x)-f(x))<p(x, x-f(x))$.

Then there exists an $x_{0} \in K$ such that $f\left(x_{0}\right) \cap g\left(x_{0}\right) \neq \varnothing$. 
Proof. Define $h: K \rightarrow \mathscr{X}(E)$ by $h(x)=x+f(x)-g(x)$ for all $x \in K$. Then $h$ is upper semicontinuous by Propositions 2.1 and 2.2. Assume that for each $x \in K, f(x) \cap g(x)=\varnothing$, so that the set $S(x)=\{y \in K: p(x, y-h(x))<$ $p(x, x-f(x))\}$ is nonempty by hypothesis. Thus $S: K \rightarrow 2^{K}$. Let $x \in K, y_{1}$, $y_{2} \in S(x)$ and $t \in[0,1]$; then $p\left(x, y_{i}-h(x)\right)<p(x, x-f(x))$.for $i=1,2$. Since $t\left(y_{1}-h(x)\right)+(1-t)\left(y_{2}-h(x)\right)=t y_{1}+(1-t) y_{2}-h(x)$, and since $p(x, \cdot)$ is convex, we see that

$$
p\left(x, t y_{1}+(1-t) y_{2}-h(x)\right)<p(x, x-f(x)),
$$

so that $t y_{1}+(1-t) y_{2} \in S(x)$. Hence $S(x)$ is convex for each $x \in K$.

Now let $u \in K$. We shall show that $S^{-1}(u)$ is open in $K$. Indeed, if $x \in S^{-1}(u)$, then $u \in S(x)$, so that $p(x, u-h(x))<p(x, x-f(x))$. Let $\varepsilon=[p(x, x-$ $f(x))-p(x, u-h(x))] / 2$. Since $p$ is ultimately continuous at $(x, x-f(x))$, there exist an open neighborhood $U_{1}$ of $x$ in $K$ and an open set $G$ in $E$ with $x-f(x) \subset G$ such that $|p(y, A)-p(x, x-f(x))|<\varepsilon$ for all $(y, A) \in K \times \Omega$ with $y \in U_{1}$ and $A \subset G$. For each $a \in x-f(x)$, let $N_{a}$ be an open neighborhood of 0 in $E$ such that $a+N_{a}+N_{a} \subset G$. Since $x-f(x)$ is compact, there exist $a_{1}, \ldots, a_{n} \in x-f(x)$ such that $x-f(x) \subset \bigcup_{i=1}^{n}\left(a_{i}+N_{a_{i}}\right)$. Since $f$ is upper semicontinuous at $x$, and since $f(x) \subset x-\bigcup_{i=1}^{n}\left(a_{i}+N_{a_{i}}\right)$, which is open, there exists an open neighborhood $U_{2}$ of $x$ in $K$ such that $f(y) \subset x-$ $\bigcup_{i=1}^{n}\left(a_{i}+N_{a_{i}}\right)$ for all $y \in U_{2}$. Let $V_{1}=U_{1} \cap U_{2} \cap\left(x+\cap_{i=1}^{n} N_{a_{i}}\right)$. Then $V_{1}$ is an open neighborhood of $x$ in $K$. Let $y \in V_{1}$; as $y \in U_{2}$, we have $f(y) \subset x-$ $\cup_{i=1}^{n}\left(a_{i}+N_{a_{i}}\right)$, so that

$$
x-f(y) \subset \bigcup_{i=1}^{n}\left(a_{i}+N_{a_{i}}\right) ;
$$

as $y \in x+\bigcap_{i=1}^{n} N_{a_{i}}$, we have $y-x \in \bigcap_{i=1}^{n} N_{a_{i}}$, so that $y-f(y)=y-x+x-$ $f(y) \subset \cap_{i=1}^{n} N_{a_{i}}+\cup_{i=1}^{n}\left(a_{i}+N_{a_{i}}\right)$ by (*). It follows that

$$
y-f(y) \subset \bigcup_{i=1}^{n}\left(a_{i}+N_{a_{i}}+N_{a_{i}}\right) \subset G ;
$$

as $y \in U_{1}$, by $(* *)$, we have

$$
|p(y, y-f(y))-p(x, x-f(x))|<\varepsilon .
$$

Next, since $p$ is also ultimately continuous at $(x, u-h(x))$, there exist an open neighborhood $U_{3}$ of $x$ in $K$ and an open set $G^{\prime}$ in $E$ with $u-h(x) \subset G^{\prime}$ such that $|p(y, A)-p(x, u-h(x))|<\varepsilon$ for all $(y, A) \in K \times \Omega$ with $y \in U_{3}$ and $A \subset G^{\prime}$. Since $h(x) \subset u-G^{\prime}$, which is open, and since $h$ is upper semicontinuous at $x$, there exists an open neighborhood $U_{4}$ of $x$ in $K$ such that $h(y) \subset u-G^{\prime}$ for all $y \in U_{4}$. Let $V_{2}=U_{3} \cap U_{4}$. Then $V_{2}$ is an open neighborhood of $x$ in $K$. Let $y \in V_{2}$; as $y \in U_{4}$, we have $h(y) \subset u-G^{\prime}$, so that

$$
u-h(y) \subset G^{\prime}
$$


as $y \in U_{3}$, by $(* * *)$, we have

$$
\text { (††) } \quad|p(y, u-h(y))-p(x, u-h(x))|<\varepsilon \text {. }
$$

Let $V=V_{1} \cap V_{2}$. Then $V$ is an open neighborhood of $x$ in $K$ such that for each $y \in V,(\dagger)$ and $(\dagger)$ hold; it follows that

$$
\begin{aligned}
p(y, u-h(y)) & <p(x, u-h(x))+\varepsilon \\
& =p(x, x-f(x))-\varepsilon \\
& <p(y, y-f(y))
\end{aligned}
$$

so that $u \in S(y)$ and hence $y \in S^{-1}(u)$ for all $y \in V$. Therefore $S^{-1}(u)$ is open for each $u \in K$.

By K. Fan's Theorem, there exists an $x_{0} \in K$ such that $x_{0} \in S\left(x_{0}\right)$ ); thus we have

$$
p\left(x_{0}, g\left(x_{0}\right)-f\left(x_{0}\right)\right)=p\left(x_{0}, x_{0}-h\left(x_{0}\right)\right)<p\left(x_{0}, x_{0}-f\left(x_{0}\right)\right),
$$

which contradicts (i). This shows that there must exist an $x_{0} \in K$ such that $f\left(x_{0}\right) \cap g\left(x_{0}\right) \neq \varnothing$. This completes the proof.

By applying Theorem 2.4 and an argument similar to that used in proving Theorem 1 in [4], we obtain the following generalization of Theorem 1 in [4].

Corollary 2.5. Let $E$ be a Hausdorff topological vector space, let $K \subset E$ be nonempty compact convex and let $f, g: K \rightarrow \mathscr{K}(E)$ be upper semicontinuous. Let $\Omega \subset 2^{E}$ be convex and contain all sets of the form $x-f(x), y-x+g(x)-f(x)$, for $x, y \in K$. Suppose $p: K \times \Omega \rightarrow \mathbf{R}$ is ultimately continuous on $K \times \Omega$. Assume that

(i) $p(x, x-f(x))=p(x, g(x)-f(x))$ for all $x \in K$, and

(ii) for each $x \in K$ with $f(x) \cap g(x)=\varnothing$, there exists $y \in I_{K}(x)$ such that $p(x, y-x+g(x)-f(x))<p(x, x-f(x))$.

Then there exists an $x_{0} \in K$ such that $f\left(x_{0}\right) \cap g\left(x_{0}\right) \neq \varnothing$.

By applying Corollary 2.5 and an argument similar to that used in proving Theorem 2 in [4], we obtain the following generalization of Theorem 2 in [4].

Corollary 2.6. Let $E$ be a Hausdorff topological vector space, let $K \subset E$ be nonempty comapct convex and let $f, g: K \rightarrow \mathscr{X}(E)$ be upper semi-continuous. Let $\Omega \subset 2^{E}$ be convex and contain all sets of the form $x-f(x), y-x+g(x)-f(x)$, for $x, y \in K$. Suppose $p: K \times \Omega \rightarrow \mathbb{R}$ is ultimately continuous on $K \times \Omega$. Assume that

(i) $p(x, x-f(x))=p(x, g(x)-f(x))$ for all $x \in K$, and

(ii) for each $x \in K$ with $f(x) \cap g(x)=\varnothing$, there exist $y \in O_{K}(x)$ such that $p(x, y-x+g(x)-f(x))<p(x, x-f(x))$.

Then there exists an $x_{0} \in K$ such that $f\left(x_{0}\right) \cap g\left(x_{0}\right) \neq \varnothing$. 
Let $E$ be a locally convex topological vector space and let $p$ be any continuous seminorm on $E$. If $A, B \subset E$ are nonempty, let $d_{p}(A, B)=\inf \{p(a-b)$ : $a \in A$ and $b \in B\}$; if $A=\{a\}$, we shall write $d_{p}(a, B)$ instead of $d_{p}(\{a\}, B)$. The following result is motivated by the proof of Theorem 3.1 in [11].

LEMMA 2.7. Let $E$ be a Hausdorff locally convex topological vector space, let $K \subset E$ be nonempty comapct convex and let $f, g: K \rightarrow \mathscr{C}(E)$ be upper semicontinuous such that for each $x \in K$, either $f(x)$ or $g(x)$ is compact. Assume that for each continuous seminorm $p$ on $E$, there exists an $x \in K$ such that $d_{p}(f(x), g(x))=0$. Then there exists an $x_{0} \in K$ such that $f\left(x_{0}\right) \cap g\left(x_{0}\right) \neq \varnothing$.

Proof. Let $\mathscr{P}$ be the set of all continuous seminorms on $E$. For each $p \in \mathscr{P}$, let $K_{p}=\left\{x \in K: d_{p}(f(x), g(x))=0\right\}$. If $p \in \mathscr{P}$ is arbitrarily fixed, then $K_{p}$ is nonempty by hypothesis; we shall show that $K_{p}$ is also closed in $K$. Indeed, let $\left(x_{\alpha}\right)_{\alpha \in \Gamma}$ be a net in $K_{p}$ such that $x_{\alpha} \rightarrow x$ for some $x \in K$. Suppose $r=$ $d_{p}(f(x), g(x))>0$. Let $V_{f}=\left\{z \in E: d_{p}(z, f(x))<r / 3\right\}$ and $V_{g}=\{z \in E$ : $\left.d_{p}(z, g(x))<r / 3\right\}$. Then $V_{f}$ and $V_{g}$ are open in $E$, and $f(x) \subset V_{f}$ and $g(x) \subset V_{g}$. Since $f$ and $g$ are upper semicontinuous at $x$, there exists a neighborhood $U$ of $x$ in $K$ such that for all $y \in U, f(y) \subset V_{f}$ and $g(y) \subset V_{g}$. Since $x_{\alpha} \rightarrow x$, there exists $\alpha_{0} \in \Gamma$ such that $x_{\alpha} \in U$ for all $\alpha \geqslant \alpha_{0}$; it follows that, in particular, $f\left(x_{\alpha_{0}}\right) \subset V_{f}$ and $g\left(x_{\alpha_{0}}\right) \subset V_{g}$, so that $d_{p}\left(f\left(x_{\alpha_{0}}\right), g\left(x_{\alpha_{0}}\right)\right) \geqslant r / 3$, which contradicts our assumption that $d_{p}\left(f\left(x_{\alpha_{0}}\right), g\left(x_{\alpha_{0}}\right)\right)=0$. Thus $d_{p}(f(x), g(x))=0$, whence $x \in K_{p}$. Therefore $K_{p}$ is closed in $K$ for each $p \in \mathscr{P}$. Now let $\left\{p_{1}, \ldots, p_{n}\right\}$ be any finite subset of $\mathscr{P}$. Let $p=\sum_{i=1}^{n} p_{i}$. Then $p \in \mathscr{P}$, and $\cap_{i=1}^{n} K_{p_{i}} \supset K_{p} \neq \varnothing$. Thus the family $\left\{K_{p}: p \in \mathscr{P}\right\}$ has the finite intersection property, whence, by compactness of $K, \bigcap_{p \in \mathscr{P}} K_{p} \neq \varnothing$. It follows that there exists an $x_{0} \in K$ such that $d_{p}\left(f\left(x_{0}\right), g\left(x_{0}\right)\right)=0$ for all $p \in \mathscr{P}$. By the HahnBanach separation theorem, $f\left(x_{0}\right) \cap g\left(x_{0}\right) \neq \varnothing$. This completes the proof.

The following result generalizes part of Theorem 3 in [12]. We shall present a different proof than the one used in [12].

THEOREM 2.8. Let $E$ be a Hausdorff locally convex topological vector space, let $K \subset E$ be nonempty compact convex and let $f, g: K \rightarrow \mathscr{C}(E)$ be continuous such that for each $x \in K$, either $f(x)$ or $g(x)$ is compact. Suppose for each $x \in K$ and for each continuous seminorm $p$ on $E$ with $d_{p}(f(x), g(x))>0$, we have $d_{p}(K, x+f(x)-g(x))<d_{p}(f(x), g(x))$. Then there exists an $x_{0} \in K$ such that $f\left(x_{0}\right) \cap g\left(x_{0}\right) \neq \varnothing$. 
Proof. Define $h: K \rightarrow \mathscr{C}(E)$ by $h(x)=x+f(x)-g(x)$ for all $x \in K$. Then $h$ is lower semicontinuous on $K$ by Proposition 2.1. Let $\mathscr{P}$ be the set of all continuous seminorms on $E$. By Lemma 2.7, it is sufficient to show that for each $p \in \mathscr{P}$, there exists an $x \in K$ such that $d_{p}(f(x), g(x))=0$. If not, then there exists a $p \in \mathscr{P}$ such that $d_{p}(f(x), g(x))>0$ for all $x \in K$, so that the set $S(x)=\left\{y \in K: d_{p}(y, h(x))<d_{p}(f(x), g(x))\right\}$ is nonempty for all $x \in K$, by hypothesis. Thus $S: K \rightarrow 2^{K}$. Let $x \in K$. As $h(x)$ is convex, $d_{p}(\cdot, h(x))$ is a convex function on $K$, and hence $S(x)$ is convex. Let $u \in K$. We shall show that $S^{-1}(u)$ is open in $K$. Indeed, if $x \in S^{-1}(u)$, then $u \in S(x)$, so that $d_{p}(u, h(x))$ $<d_{p}(f(x), g(x))$. Let $\varepsilon=\left[d_{p}(f(x), g(x))-d_{p}(u, h(x))\right] / 4$. Choose $w_{0} \in h(x)$ such that $p\left(u-w_{0}\right)<d_{p}(u, h(x))+\varepsilon$. Let $G=\left\{z \in K: p\left(z-w_{0}\right)<\varepsilon\right\}$. Then $G$ is open in $K$, and $G \cap h(x) \neq \varnothing$. Since $h$ is lowe semicontinuous at $x$, there exists an open neighborhood $V_{1}$ of $x$ in $K$ such that $h(y) \cap G \neq \varnothing$ for all $y \in V_{1}$. Let $V_{2}=V_{1} \cap\{z \in K: p(z-x)<\varepsilon\}$. Then $V_{2}$ is an open neighborhood of $x$ in $K$. Let $y \in V_{2}$. Then $h(y) \cap G \neq \varnothing$, and if we choose any $w \in h(y) \cap G$, we have

$$
\begin{aligned}
d_{p}(u, h(y)) & \leqslant p(u-w) \leqslant p\left(u-w_{0}\right)+p\left(w_{0}-w\right) \\
& <d_{p}(u, h(x))+\varepsilon+\varepsilon=d_{p}(u, h(x))+2 \varepsilon .
\end{aligned}
$$

Next, note that for $V_{f}=\left\{z \in K: d_{p}(z, f(x))<\varepsilon / 2\right\}$ and $V_{g}=\{z \in K$; $\left.d_{p}(z, g(x))<\varepsilon / 2\right\}, V_{f}$ and $V_{g}$ are open in $K$, and they contain $f(x)$ and $g(x)$, respectively. Since $f$ and $g$ are upper semicontinuous at $x$, there exists an open neighborhood $V_{3}$ of $x$ in $K$ such that $f(y) \subset V_{f}$ and $g(y) \subset V_{g}$ for all $y \in V_{3}$. Let $y \in V_{3}$, and then choose $a \in f(y)$ and $b \in g(y)$ such that $p(a-b)<$ $d_{p}(f(y), g(y))+\varepsilon$. Since $a \in f(y) \subset V_{f}$ and $b \in g(y) \subset V_{g}$, there are $a_{0} \in f(x)$ and $b_{0} \in g(x)$ with $p\left(a-a_{0}\right)<\varepsilon / 2$ and $p\left(b-b_{0}\right)<\varepsilon / 2$. It follows that

$$
\begin{aligned}
d_{p}(f(x), g(x)) & \leqslant p\left(a_{0}-b_{0}\right) \\
& \leqslant p\left(a_{0}-a\right)+p(a-b)+p\left(b-b_{0}\right) \\
& <\frac{\varepsilon}{2}+d_{p}(f(y), g(y))+\varepsilon+\frac{\varepsilon}{2} \\
& =d_{p}(f(y), g(y))+2 \varepsilon .
\end{aligned}
$$

If now $V=V_{2} \cap V_{3}$, then $V$ is an open neighborhood of $x$ in $K$, and for each $y \in V$, we have

$$
\begin{aligned}
d_{p}(u, h(y)) & <d_{p}(u, h(x))+2 \varepsilon, \text { by }(*) \\
& =d_{p}(f(x), g(x))-2 \varepsilon \\
& <d_{p}(f(y), g(y)), \quad \text { by }(* *)
\end{aligned}
$$


so that $u \in S(y)$, and hence $y \in S^{-1}(u)$ for all $y \in V$. Therefore $S^{-1}(u)$ is open in $K$ for each $u \in K$. By $K$. Fan's Theorem, there exists an $x_{0} \in K$ such that $x_{0} \in S\left(x_{0}\right)$, so that $d_{p}\left(x_{0}, h\left(x_{0}\right)\right)<d_{p}\left(f\left(x_{0}\right), g\left(x_{0}\right)\right)$, and this is impossible because $d_{p}\left(x_{0}, h\left(x_{0}\right)\right)=d_{p}\left(f\left(x_{0}\right), g\left(x_{0}\right)\right)$. This completes the proof.

Analogous to Corollary 2.5 and Corollary 2.6, we have the following results, which form generalizations of Corollary 1 (respectively, Corollary 1') in [4].

COROLlaRY 2.9. Let $E$ be a Hausdorff locally convex topological vector space, let $K \subset E$ be nonempty compact convex and let $f, g: K \rightarrow \mathscr{C}(E)$ be continuous such that for each $x \in K$, either $f(x)$ or $g(x)$ is compact. Suppose for each $x \in K$ and for each continuous seminorm $p$ on $E$ with $d_{p}(f(x), g(x))>0$, there exist $y \in I_{K}(x)$ (respectively, $\left.y \in O_{K}(x)\right)$ such that $d_{p}(y, x+f(x)-g(x))<d_{p}(f(x), g(x))$. Then there exists an $x_{0} \in K$ such that $f\left(x_{0}\right) \cap g\left(x_{0}\right) \neq \varnothing$.

The following is an immediate consequence of Corollary 2.9.

COROLlARY 2.10. Let E be a Hausdorff locally convex topoological vector space, let $K \subset E$ be nonempty compact convex and let $f, g: K \rightarrow \mathscr{C}(E)$ be continuous such that for each $x \in K$, either $f(x)$ or $g(x)$ is compact. Suppose for each $x \in K$ and for each continuous seminorm $p$ on $E$ with $d_{p}(f(x), g(x))>0$, there exists $y \in$ $\operatorname{cl}\left(I_{K}(x)\right)$ (respectively, $y \in \operatorname{cl}\left(O_{K}(x)\right)$ such that $d_{p}(y, x+f(x)-g(x))<$ $d_{p}(f(x), g(x))$. Then there exist an $x_{0} \in K$ such that $f\left(x_{0}\right) \cap g\left(x_{0}\right) \neq \varnothing$.

\section{References}

[1] C. Berge, Topological Spaces (Oliver \& Boyd, Edinburgh and London, 1963).

[2] F. E. Browder, A new generalization of the Schauder fixed point theorem, Math. Ann. 174 (1967), 285-290.

[3] F. E. Browder, The fixed point theory of multi-valued mappings in topological vector spaces, Math. Ann. 177 (1968), 283-301.

[4] F. E. Browder, On a sharpened form of the Schauder fixed-point theorem, Proc. Nat. Acad. Sci. U. S. A. 74 (1977), 4749-4751.

[5] J. Caristi, Fixed point theorem for mapping satisfying inwardness conditions, Trans. Amer. Math. Soc. 215 (1976), 241-251.

[6] K. Fan, A generalization of Tychonoff's fixed point theorem, Math. Ann. 142 (1961), 305-310.

[7] K. Fan, Extensions of two fixed point theorems of F. E. Browder, Math. Z. 112 (1969), 234-240.

[8] B. Halpern and G. Bergman, A fixed point theorem for inward and outward maps, Trans. Amer. Math. Soc. 130 (1968), 353-358.

[9] J. H. Jiang, Fixed point theorems for multi-valued mappings in locally convex spaces, Acta Math. Sinica 25 (1982), 365-373. 
[10] J. H. Jiang, Generalization of two fixed point theorems of S. Reich, Acta Math. Sinica 24 (1981), 359-364.

[11] S. Reich, Approximate selections, best approximations, fixed points, and invariant sets, $J$. Math. Anal. Appl. 62 (1978), 104-113.

[12] S. Reich, Fixed point theorems for set-valued mappings, J. Math. Anal. Appl. 69 (1979), 353-358.

[13] S. Reich, Some problems and results in fixed point theory, Contemporary Math. 21 (1983), 179-187.

Department of Mathematics, Statistics and Computing Science

Dalhousie University

Halifax, Nova Scotia B3H 3J5

Canada 